\title{
Ambientes restauradores no trânsito: variabilidade da freqüuência cardíaca e tempo de reação*
}

\author{
Adilson André Martins Monte ${ }^{l}$ \\ Jéferson Passig ${ }^{2}$ \\ Emílio Takase ${ }^{3}$ \\ Ariane Kuhnen ${ }^{4}$ \\ Universidade Federal de Santa Catarina
}

Neste trabalho investigou-se a aplicação da variabilidade da freqüência cardíaca (VFC) para a monitorização e gravação dos estados de excitação autonômica de um motorista em uma viagem interestadual de 725 $\mathrm{km}$. O sinal de VFC é usado como uma ferramenta para a avaliação do sistema nervoso no controle da freqüência cardíaca. Levaramse em consideração as variáveis ambientais decorrentes desta tarefa. Em cinco momentos distintos da viagem foram medidos os sinais R-R da freqüência ventricular (intervalo entre duas ondas $\mathrm{R}$ corresponde à freqüência de despolarização ventricular) e realizados teste de tempo de reação do motorista. Os resultados expressaram fortes correlações entre as variáveis de baixa freqüência da variabilidade da freqüência cardíaca (LF) e alta freqüência da variabilidade da freqüência cardíaca (HF) com o tempo de reação, o que indica a necessidade de investir numa possível monitoração contínua da VFC do motorista como indicador da sua condição de dirigir. Ferramenta que colaboraria na prevenção contra acidentes de trânsito.
This work investigated the application of heart rate variability (HRV) for monitoring and recording the states of autonomic arousal of a driver in interstate travel a $725 \mathrm{~km}$ away. The HRV signal is used as a tool for assessing the performance of the nervous system in heart rate control. They took into account environmental variables resulting from this task. At five different moments of the trip were measured signals R-R of ventricular rate (interval between two $R$ waves matches the frequency of ventricular depolarization) and simultaneously performed a reaction time test driver. The results expressed strong correlations among the low-frequency heart rate variability (LF - Low Frequency) and high frequency heart rate variability (HF - High Frequency) with the reaction time, which indicates the need to invest in a possible continuous monitoring of HRV the driver as an indicator of his condition to drive. Tool that would work in preventing traffic accidents.

* Environments restorers in traffic: heart rate variability and reaction time

${ }^{1}$ Doutorando do Programa de Pós-Graduação em Psicologia, Universidade Federal de Santa Catarina, (adilsomonte@gmail.com).

${ }^{2}$ Mestrando do Programa de Pós-Graduação em Psicologia, Universidade Federal de Santa Catarina, (jpassig@bol.com.br).

${ }^{3}$ Doutor em Psicologia Experimental, Professor do Programa de Pós-Graduação em Psicologia, Universidade Federal de Santa Catarina.

${ }^{4}$ Doutora em Ciências Humanas, Professora do Programa de Pós-Graduação em Psicologia, Universidade Federal de Santa Catarina. Endereço para correspondências: Universidade Federal de Santa Catarina, Centro de Filosofia e Ciências Humanas, Departamento de Psicologia, Laboratório de Psicologia Ambiental, Florianópolis, SC, 88040-970. 


\section{HUMANAS}

Sugere-se a utilização de ambientes restauradores nas estradas a fim de evitar acidentes desse gênero.

Palavras-chave: PsicologiaAmbiental-Ambientes restauradores - Trânsito - Variabilidade de freqüência cardíaca-Tempo de reação
We suggest the use of restorative environments on the road to avoid accidents of this kind.

Keywords: Environmental Psychology Restorative environments - Traffic - Heart rate variability - Reaction time

\section{Introdução}

Z ste artigo relaciona quatro temas distintos e específicos: trânsito, - ambientes restauradores, variabilidade da freqüência cardíaca e seus componentes e o tempo de reação. E podem se correlacionar na medida em que podem dar indicações de como melhorar o uso das estradas, que atualmente se tornaram sinônimas de perigo a todos que as utilizam. Assim, dar ênfase a estas questões é procurar ultrapassar as demarcações teóricas, buscando indicar medidas que desdobrem em resultados efetivos.

\subsection{O trânsito}

Dados fornecidos pela Polícia Rodoviária Federal Brasileira mostram que mais de $90 \%$ dos acidentes são causados por erros humanos associados a cansaço, distração, imprudência e estresse. $\mathrm{O}$ ambiente propiciado pelo ato de dirigir também influencia fortemente o comportamento o motorista. "Os engarrafamentos estimulam comportamentos de raiva e agressividade que, por sua vez, se associam ao comportamento infrator de trânsito", alerta o psicólogo Fábio Henrique Vieira de Cristo Silva em entrevista para o Jornal da Universidade (Março, Ano XII, Número 116, UFRGS).

Em um estudo que procurou investigar a relação entre comportamentos inadequados de motoristas de ônibus no Distrito Federal, Abelardo Vinagre da Silva na sua dissertação de mestrado defendida na UnB em fevereiro de 2008 após observações sistemáticas durante 73 viagens urbanas realizadas por um mesmo motorista indicaram uma correlação positiva entre a freqüência de comportamentos inadequados e o tempo despendido nas viagens (GÜNTHER, 2004).

Segundo Rocha (2005; p. 15): "a engenharia tem fornecido aos veículos formidáveis avanços tecnológicos e equipado as vias com sofisticados recursos" (onde os governos permitem), com a preocupação de proporcionar melhores condições de fluxo e segurança da crescente frota de veículos e dos usuários do trânsito. Por outro lado, a reflexão em torno dos erros e violações no trânsito e das variáveis que os antecedem, determinam ou influenciam, vêm ganhando maior dimensão nos debates entre os estudiosos. 
Quando comparados aos avanços tecnológicos de equipamentos e veículos, as pesquisas e os avanços técnicos nas questões comportamentais no trânsito não acontecem na mesma velocidade devido às naturais resistências humanas e à própria natureza do assunto. Descompasso este também responsável talvez pelas dificuldades do trânsito nas grandes cidades, ao lado de outros problemas sociais.

As jornadas de trabalho de um motorista de ônibus no Brasil ode chegar a 12 horas (OLIVEIRA, 2003). A realização de horas-extras por motoristas de transporte urbano de passageiros em outro período da jornada de trabalho aumenta a probabilidade de ocorrer acidentes de trânsito, tendo em vista os eventuais desgastes físicos e psicológicos relacionados ao cotidiano de trabalho dos motoristas (GARCIA et al., 2005).

Continua sendo primordial o investimento em pesquisas relativas à Psicologia Aplicada ao Trânsito, sendo necessários estudos sobre a validade preditiva dos instrumentos em relação a critérios de segurança, com delineamento que incluam, além de uma grande quantidade de amostra, uma descrição verdadeiramente representativa da população brasileira. Desta forma poder-se-ia, clarificar a relação entre aspectos psicológicos (personalidade, habilidades e inteligência) e o comportamento do motorista (SILVA, 2008). A concepção de acidentes de trânsito em termos de epidemia responsabiliza-nos a tornar o trânsito mais seguro para os participantes.

\subsection{Psicologia Ambiental e ambientes restauradores}

A Psicologia Ambiental estuda a relação recíproca entre pessoas e o ambiente físico, construído e/ou natural (GÜNTHER, 2004). Considerar isto leva a compreendermos o ambiente não como fragmentos de territórios presente em algum lugar qualquer. Mas sim como uma síntese organizada pela pessoa no referido espaço, herança do passado que se expõe no presente (TASSARA, 2005). É disto que fala Giuliani (2005), Marçolla (2002) e Uzzell (2005) quando apontam a necessidade de uma abordagem "contextual". As partes do ambiente a serem consideras como contexto podem variar segundo a compreensão do pesquisador, mas a ação humana é algo que sempre ocorre inserida em algum ambiente. As pessoas devem ser analisadas em seus contextos sociais e ambientais específicos, que por sua vez estão dentro de um contexto social maior.

Dentro desse horizonte de compreensão, uma forte tendência de estudos na área tem sido o tema dos "ambientes restauradores" no sentido de que podem contribuir para reduzir o estresse e promover o humor e sentimentos positivos nas pessoas (GIULIANI \& SCOPELLITI, 2004; VELARDE et al., 2007), proporcionando uma experimentação de bem-estar (CONSTANTINO, 2004). 


\section{HUMANAS}

Ambientes que tem a capacidade de propiciar "o sentido e a identidade, situando o indivíduo social, econômica e culturalmente" (MOSER, 2005a; p. 282). Pesquisas empíricas, tais como as de Ivarsson e Hagerhall (2008a), Velarde e colaboradores (2007), Berg (2007), Joye (2007) e Kaplan (2001), comprovam os benefícios, acrescentando que em termos de saúde o contato com ambientes restauradores alivia o estresse físico e psicológico e, a longo prazo, há uma melhora significativa na saúde global. A partir da década de 1980 revigorou um crescente interesse de pesquisas científicas buscando compreender os efeitos na saúde humana do ambiente físico e social. Por exemplo, médicos passaram a considerar que a recuperação de um paciente cardíaco pode estar relacionada ao convívio dele em ambientes verdes, tais como praças e parques. Desde então, os espaços abertos, seja em hospitais ou urbanos, vem sendo mais e mais considerados para a promoção do bem-estar e no desenvolvimento e manutenção da saúde. Um dos pioneiros no desenvolvimento teórico contemporâneo foi Ulrich (1984), que buscou demonstrar serem os ambientes naturais redutores do estresse em comparação com ambientes urbanos comuns. Outras pesquisas também tem se ocupado com este tema (BERG et al., 2010a, 2010b; HARTIG et al., 1996b; KARMANOV \& HAMEL 2008). Outro marco histórico importante na questão da restauração foi construído por Rachel e Stephen Kaplan, onde descrevem os efeitos de ambientes naturais enquanto restauradores na recuperação da fadiga psicológica (KAPLAN \& KAPLAN, 1989).

É necessário compreender a questão da restauração como um recorte feito no contexto sócio-histórico-material em que a pessoa está inserida (EVANS, 2005; GIULIANI \& SCOPELLITI, 2004; VALERA, 1996). Entretanto há lacunas do conhecimento que requerem mais investigação, pois Velarde e colaboradores (2007) questionam quais são as qualidades de uma paisagem para ser reparadora. $\mathrm{O}$ autor enfatiza que identificar estas qualidades, a fim de aplicá-las ao projeto paisagístico de uma determinada cidade é um dos principais desafios às futuras pesquisas.

Kaplan (1983) destaca que muitos elementos disponíveis no ambiente são muitas vezes ignorados na análise da adaptação ou congruência entre a pessoa e o ambiente. Para Kort e colaboradores (2006) os ambientes restauradores são ambientes que podem ajudar na restauração dos recursos de atenção esgotados ou reduzir os estresses emocionais e psicossomáticos.

Numa pesquisa realizada com 18 pessoas, Kjellgren e colaboradores (2010) compararam os efeitos de restauração através do relaxamento de 30 minutos em um ambiente natural através da simulação do próprio ambiente natural. Os participantes da pesquisa apresentavam queixas de estresse e/ou síndrome do esgotamento profissional. A restauração nos ambientes naturais obteve resultados eficientes. 
Conclui-se que admitir que a relação pessoa-ambiente como possibilidade restauradora é de grande importância, sobretudo na atualidade, onde determinadas dinâmicas do cotidiano expõem as pessoas à constante pressão. Assim, proporcionar ambientes que permitam que a pessoa tome distância de tais pressões é dar condições para a promoção do seu bem-estar e da sua saúde.

\subsection{Variabilidade da Freqüência Cardíaca}

O sistema nervoso autônomo (SNA) desempenha um papel importante na regulação dos processos fisiológicos do organismo humano tanto em condições normais quanto patológicas. Dentre as técnicas utilizadas para sua avaliação, a variabilidade da freqüência cardíaca (VFC) tem emergido como uma medida simples e não-invasiva dos impulsos autonômicos, representando um dos mais promissores marcadores quantitativos do balanço autonômico. A VFC descreve as oscilações no intervalo entre batimentos cardíacos consecutivos (intervalos R-R), assim como oscilações entre freqüências cardíacas (FC) instantâneas consecutivas. Trata-se de uma medida que pode ser utilizada para avaliar a modulação do SNA sob condições fisiológicas, tais como em situações de vigília e sono, diferentes posições do corpo, treinamento físico, e também em condições patológicas (VANDERLEI et al., 2009).

Segundo Novais e colaboradores (2004) e Godoy e colaboradores (2005), a VFC decompõem-se em componentes oscilatórios fundamentais, sendo que os principais são:

a) Componente de alta freqüência (High Frequency - HF), com variação de 0,15 a $0,4 \mathrm{~Hz}$, que corresponde à modulação respiratória e é um indicador da atuação do nervo vago sobre o coração;

b) Componente de baixa freqüência (Low Frequency - LF), com variação entre 0,04 e $0,15 \mathrm{~Hz}$, que é decorrente da ação conjunta dos componentes vagal e simpático sobre o coração, com predominância do simpático;

c) Componentes de muito baixa freqüência (Very Low Frequency - VLF) e ultra baixa freqüência (Ultra Low Frequency - ULF) - Índices menos utilizados cuja explicação fisiológica não está bem estabelecida e parece estar relacionada ao sistema renina-angiotensina-aldosterona, à termorregulação e ao tônus vasomotor periférico.

A relação LF/HF reflete as alterações absolutas e relativas entre os componentes simpático e parassimpático do SNA, caracterizando o balanço simpato-vagal sobre o coração (NOVAIS et al., 2004). 


\section{HUMANAS}

Para obtenção dos valores através da análise no domínio de freqüência da variabilidade da freqüência cardíaca (índices espectrais), o tacograma ${ }^{5}$ de freqüência sofre processamento matemático, gerando um gráfico que expressa a variação dos intervalos $\mathrm{R}-\mathrm{R}$ em função do tempo. $\mathrm{O}$ tacograma contém um sinal que oscila no tempo e que é processado por algoritmos matemáticos, como a transformada rápida de Fourier $(\mathrm{FFT})^{6}$ ou modelos auto-regressivos (AUBERT, 2003).

O método FFT é utilizado para obter uma estimativa de potência espectral da VFC durante fases estacionárias do experimento com a finalidade de permitir comparações entre os resultados de estudos (AUBERT, 2003).

Para análise dos índices de VFC por meio de métodos lineares vários softwares podem ser utilizados, dentre eles, o software HRV analysis (NISKANEN, 2004), o qual pode ser obtido gratuitamente pela Internet. O software Kubios HRV version 2.0 desenvolvido pelo Departament Physics University of Kuopio (Finland) igualmente desempenha esta função e também pode ser obtido de forma gratuita na Internet. Este último foi utilizado por esta pesquisa.

\subsection{Tempo de Reação}

O tempo de reação é definido como o tempo que decorre entre um estímulo recebido e uma ação realizada em decorrência deste estímulo. Nas tarefas muito simples, os tempos de reação refletem mais os processos sensório-motores do que processos cognitivos de tratamento de informação (LINDLEY et al., 1995).

Conforme Schmidt e colaboradores (2001), o tempo de reação apresenta três classificações:

1. Tempo de reação simples: é o tempo de reação mais curto que representa o tempo que decorre da apresentação de um estímulo não antecipado ao início da resposta;

2. Tempo de reação de escolha: é o intervalo de tempo entre a apresentação de um dos vários estímulos possíveis não antecipados e o começo de uma das várias respostas possíveis;

3. Tempo de reação de discriminação: é o intervalo de tempo entre a apresentação de um dos vários estímulos possíveis não antecipados e o começo de uma resposta possível.

\footnotetext{
Gráficos onde estão representados por pontos os intervalos entre os intervalos da freqüência cardíaca em um determinado período.

${ }^{6}$ A Transformada rápida de Fourier (FFT) é um algoritmo eficiente para a resolução de equações diferenciais parciais que expressa uma determinada função em termos de funções de base sinusoidal, ou seja,a função é representada por uma soma ou integral de funções sinusoidais multiplicadas por suas relativas amplitudes (coeficientes).
} 
Neste estudo o Tempo de reação (TR) medido e tomado como uma das variáveis dependentes do experimento foi o tempo de reação simples. Uma forma de simulação de uma eventual forma de ação de um motorista na sua tarefa de controlar o automóvel. A tabela 1 exemplifica a importância desta variável neste processo, principalmente no que se refere a acidentes de trânsito. Um automóvel trafegando a $120 \mathrm{~km} / \mathrm{h}$ percorre a distância de 16,7 metros a cada meio segundo $(0,5 \mathrm{~s})$.

\section{Tabela 1}

Exemplo de cálculo da distância percorrida pelo automóvel levando em relação a velocidade do mesmo e o tempo de retardo do motorista para frear o veículo.

\begin{tabular}{ccccc}
\hline TR/Vel & $\mathbf{1 2 0} \mathbf{k m} / \mathbf{h}$ & $\mathbf{8 0 k m} / \mathbf{h}$ & $\mathbf{6 0 k m} / \mathbf{h}$ & $\mathbf{4 0 k m} / \mathbf{h}$ \\
\hline $\mathbf{0 , 5 s}$ & $16,7 \mathrm{~m}$ & $11,1 \mathrm{~m}$ & $8,33 \mathrm{~m}$ & $5,55 \mathrm{~m}$ \\
$\mathbf{0 , 3 s}$ & $10 \mathrm{~m}$ & $6,67 \mathrm{~m}$ & $5 \mathrm{~m}$ & $1,11 \mathrm{~m}$ \\
\hline
\end{tabular}

\section{Material e Métodos}

\subsection{Procedimentos}

Este manuscrito relata uma pesquisa experimental que teve como variável independente a distância percorrida assim como as condições ambientais, ou seja, as condições de tempo da viagem e como variáveis dependentes a VFC e o TR.

Os dados foram coletados em uma única viagem a partir de um motorista do sexo masculino, saudável, com quarenta e três anos de idade e com experiência de direção automotiva de vinte e cinco anos. O motorista conhecia profundamente a rota traçada na viagem.

A instrumentação utilizada para a coleta é especificada a seguir conforme a sua função:

VFC - os sinais de freqüência cardíaca (R-R) foram captados através de um monitor de freqüência cardíaca da marca polar e modelo vantage $N V$. Estes dados foram transferidos para um computador pessoal através da interface polar advantage (software version 2:10:009).

TR - o software foi confeccionado em Visual Basic 5.0 pelo autor desta pesquisa e como característica de funcionamento foi programado para emitir um sinal visual em um tempo aleatório gerado entre um e dez segundos. (conforme exemplificados e ilustrado nas figuras 1 e 2).

Após coletados, os dados sofreram tratamento estatístico no software Microsoft Excel versão 2007. 


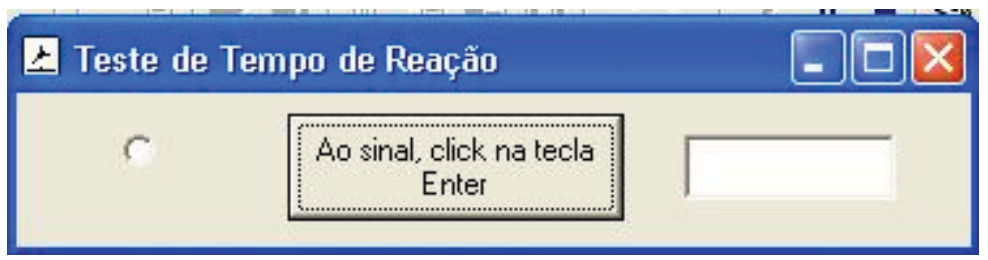

Figura 1

Tela do software do teste de reação em modo de espera.

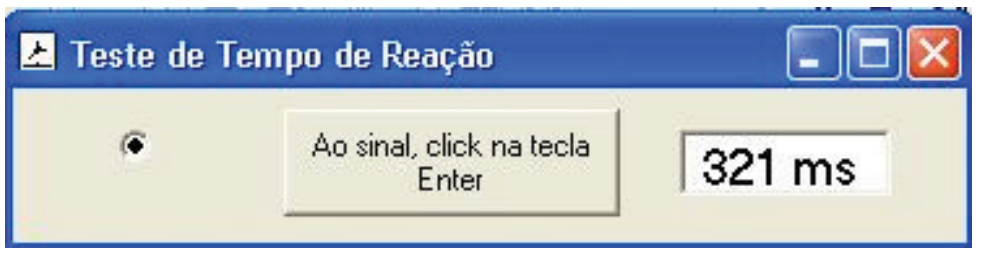

Figura 2

Tela do software do teste de reação logo após o momento do seu acionamento.

O veículo utilizado foi um Stilo da marca Fiat, modelo 2005 equipado com ar condicionado, o qual esteve em funcionamento durante toda a viagem, mantendo a temperatura em 23 graus Celsius no seu interior.

As coletas foram feitas sempre na chegada a postos de reabastecimentos de combustíveis existentes na estrada.

Cada coleta durou cinco minutos. Neste período os sinais de FC foram adquiridos continuamente e foram realizados em média, quarenta e cinco testes de TR. As coletas forma feitas em cinco etapas:

1- $0 \mathrm{Km}$ - 07:28:06 (horário de Brasília) - Começo da viagem no município de Florianópolis (SC). Motorista descansado e bastante concentrado. Tempo nublado, porém não chovia;

2- 153 km - 10:00:24 (horário de Brasília) - Município de Tubarão(SC). Tempo instável, chovia moderadamente;

3- 369 km - 12:35:12 (horário de Brasília) - Município de Osório(RS). Tempo instável, chovia moderadamente;

4- 480 km - 13:56:15 (horário de Brasília) - Município de Eldorado do Sul (RS). Tempo instável, chovia fortemente, sendo que no percurso feito na Free-way houve várias aquaplanagens do veiculo;

5- $715 \mathrm{~km}$ - 16:39:16 (horário de Brasília) - Município de Pelotas (RS). Tempo instável com chuva moderada; 
Ambientes restauradores no trânsito: variabilidade da freqüência cardíaca e tempo de reação A.A.M. Monte, J. Passig, E. Takase \& A.Kuhnen

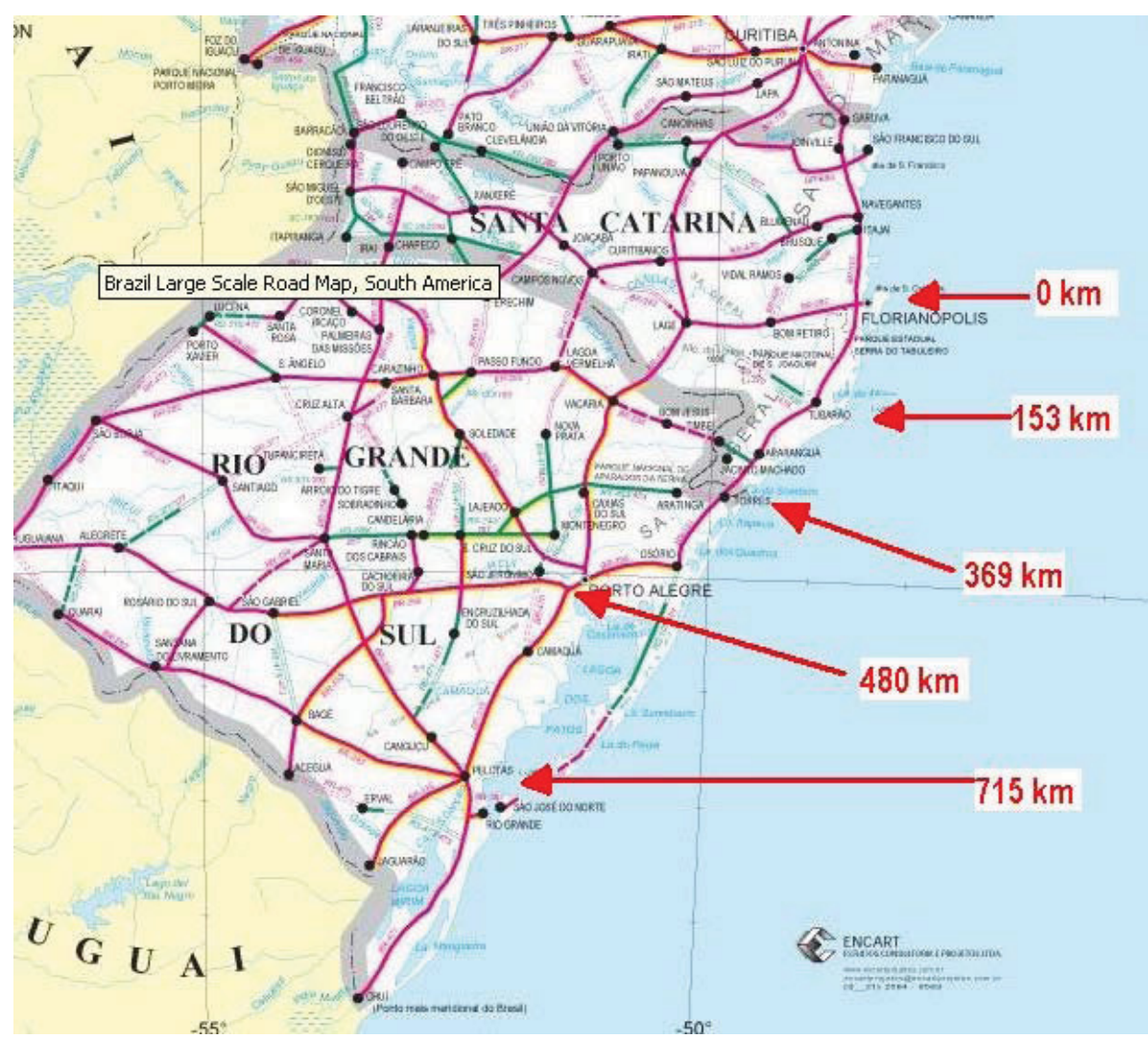

Figura 3

Rota com a localização das paradas para a coleta de dados.

\section{Resultados}

A tabela 2 expõe as médias aritméticas do TR, do desvio padrão (DP) e da relação $\mathrm{LF} / \mathrm{HF}$ (VFC):

\section{Tabela 2}

Média aritmética e DP do TR e da relação LF/HF (VFC).

\begin{tabular}{lccccc}
\hline & $\mathbf{0 ~} \mathbf{~ k m}$ & $\mathbf{1 5 3} \mathbf{~ k m}$ & $\mathbf{3 6 9} \mathbf{~ k m}$ & $\mathbf{4 8 0} \mathbf{~ k m}$ & $\mathbf{7 1 5} \mathbf{~ k m}$ \\
\hline Média TR (ms) & 391,97 & 449,83 & 526,13 & 495,62 & 515,18 \\
D.P. TR (ms) & 150,03 & 124,81 & 206,56 & 217,01 & 300,34 \\
LF/HF & 1.681 & 1.302 & 0,729 & 0,743 & 0,658 \\
\hline
\end{tabular}


Da análise dos dados resultou uma correlação entre a média do TR e LF/ HF (VFC):

$$
\tilde{\mathrm{n}}(\mathrm{TR} \text { e LF/HF })=-0,96
$$

Sendo a correlação definida como:

$$
\tilde{n}_{x, y}=\operatorname{Cov}(X, Y) /\left(\dot{o}_{x} o_{y}\right)
$$

Onde: $\mathrm{X}$ e Y são as médias das amostras; Cov é a covariância; ó $_{X} \mathrm{e} \mathrm{o}_{Y}$ os desvios padrões das amostras e $\tilde{n}_{X, Y}$ o coeficiente de correlação.

Figuras obtidas através dos relatórios fornecidos pelo software Kubios $H R V$ version 2.0 são apresentadas a seguir. As figuras correspondem a cada etapa da coleta e ilustram as relações LF/HF obtidas a partir da VFC. O componente de alta freqüência (HF) é representado pela cor mais clara e o componente de baixa freqüência (LF) pela cor mais escura.

Figura 4

0 km - Florianópolis (SC)

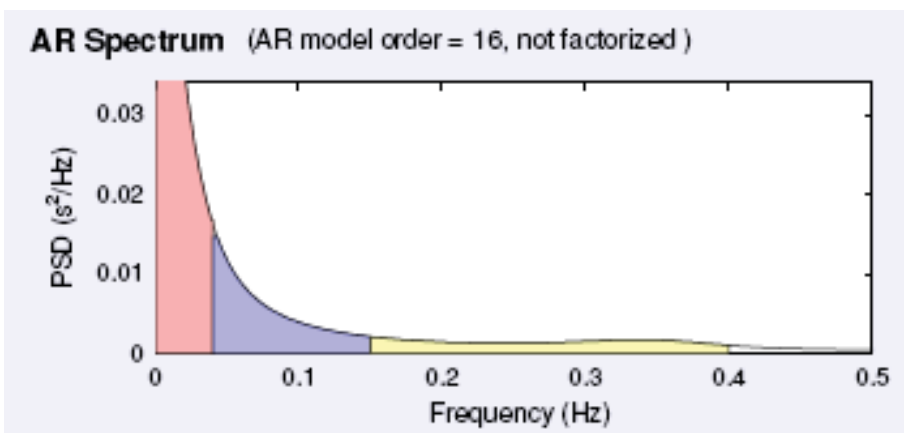

Figura 5

153 km - Tubarão(SC)

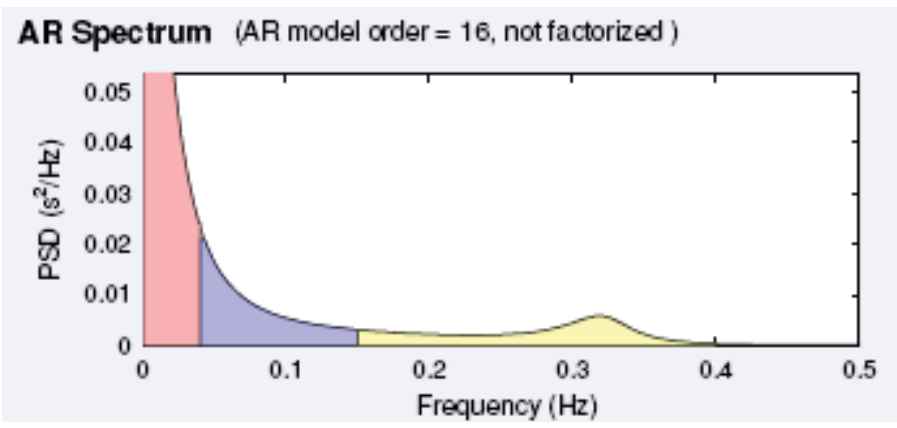


Ambientes restauradores no trânsito: variabilidade da freqüência cardíaca e tempo de reação A.A.M. Monte, J. Passig, E. Takase \& A.Kuhnen

Figura 6

369 km-Osório (RS)

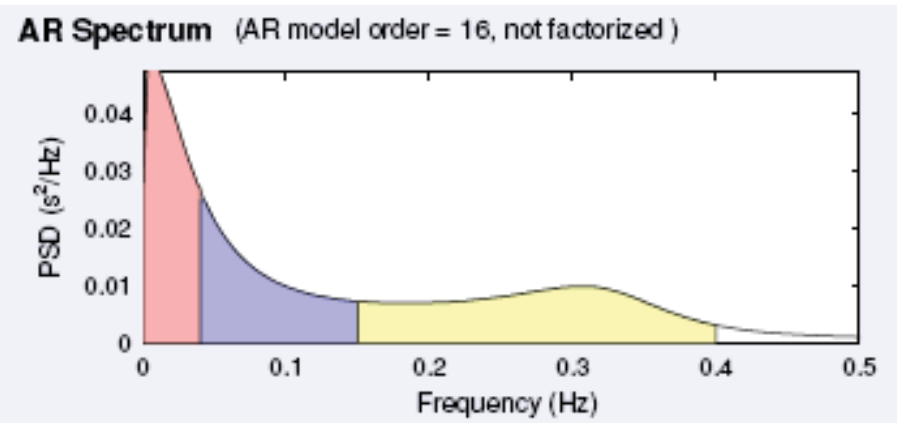

Figura 7

$480 \mathrm{~km}$ - Eldorado do Sul (RS)

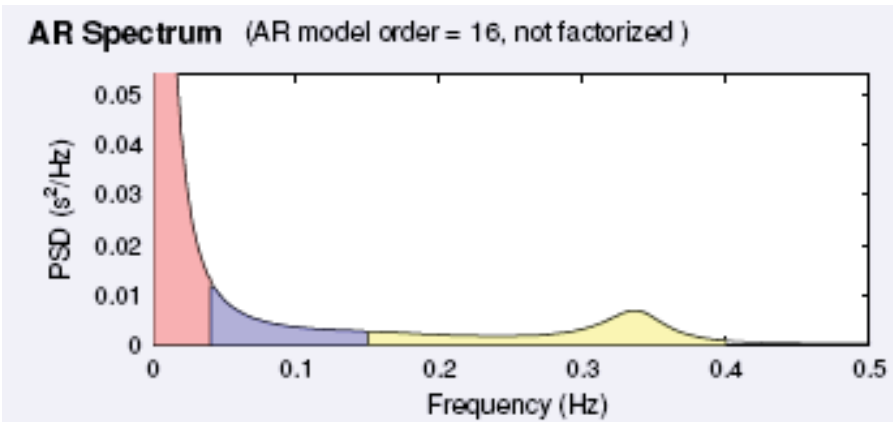

Figura 8

$715 \mathrm{~km}-$ Pelotas (RS)

AR Spectrum (AR model order $=16$, not factorized )

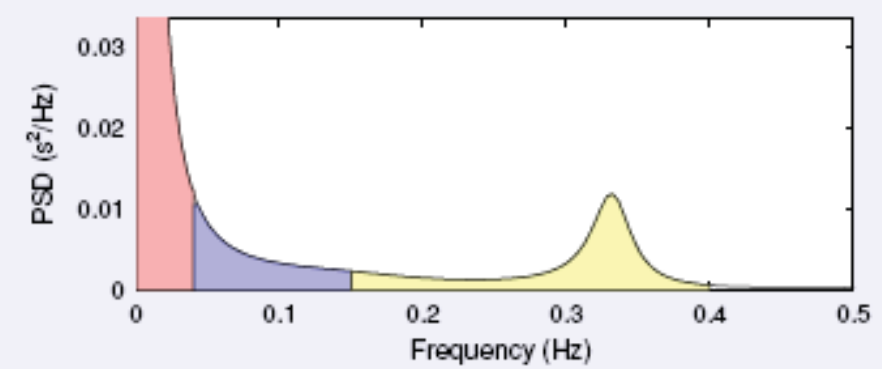




\section{HUMANAS}

\section{Discussão}

A razão LF/HF indica o estado de excitação do motorista. Quanto maior este índice, maior a excitação. Os resultados desta pesquisa indicaram uma correlação negativa forte entre LF/HF com o TR do motorista. Quanto maior o tempo de reação menor a razão $\mathrm{LF} / \mathrm{HF}$, ou seja, menor a excitação ou pior a desempenho de um motorista na sua tarefa.

Como a tabela 2 demonstra, o desempenho do motorista (TR) foi diminuindo conforme o progresso da viagem. Uma exceção ocorreu no trecho em que houve um estímulo externo (chuva intensa). Esta situação promoveu um comprometimento com a atenção do motorista (figura 7).

\section{Sugestões e considerações finais}

Faz-se indispensável adotar ações a fim de contribuir para a diminuição de acidentes que ocorrerem nas nossas estradas. Cada área de pesquisa deveria colaborar com a mudança desta realidade. Define-se a realidade como um sistema de relações, no interior do qual a pessoa é uma das partes constitutivas como integrante ativo, em que as relações que estabelece com as características ambientais apontam para uma existência de uma interdependência da pessoa e do meio (KUHNEN, 2009).

No trânsito, mais especificamente na "realidade" apontada por esta pesquisa em que o motorista foi submetido a nove horas de viagem, o sujeito, no caso o próprio motorista, é o integrante ativo do processo. Um dos caminhos para a mudança desta realidade (desastres e acidentes automobilísticos) pode estar no investimento de um "treinamento" de suas performances como o exemplo proposto a seguir.

Assim como as características biológicas e ambientais podem contribuir na mudança no desempenho das pessoas, alterando as funções cognitivas e emocionais, o treino com alguns jogos de videogame também têm mostrado a eficiência na melhora da concentração (atenção: foco interno e externo), nas estratégias de aprendizagem, no controle da ansiedade, entre outras habilidades cognitivas e emocionais (TAKASE, 2009). Porém, aliado a este tipo de atitude, poder-se-ia também investir em tecnologia aplicada ao objeto (automóvel).

A pesquisa de Riener e colaboradores (2009) estabelece contornos para este rumo. Eles investigaram as relações entre VFC e os estados emocionais de motoristas. Para isso, monitoraram motoristas em diferentes trechos de vinte quilômetros numa região central da cidade austríaca de Linz. Pela manhã e à tarde, trechos com congestionamentos ou com tráfego rápido. Enfim, obtiveram relações entre os estados de excitação do motorista e determinadas regiões. 
Sugerem além de um maior esforço no sentido de adquirir mais dados dos ambientes específicos de trânsito, uma indicação de regiões "estressantes" a partir desta apropriação de conhecimento. Colaborando com o motorista através de informações como uma espécie de mapeamento emocional do trânsito de determinada região.

Com métodos diferentes, porém com objetivos parecidos (auxiliar o motorista através de informações) caminhamos na mesma linha de ação, ou seja, implementar tecnologia de apoio à prevenção.

Ao encontrar uma correlação forte entre LF/HF com o TR do motorista, pode-se propor uma monitoração contínua do mesmo com o propósito de indicar situações de condições adversas à tarefa de dirigir um automóvel. Esta pesquisa levanta a possibilidade desta monitoração sem qualquer interferência durante o processo primário.

Sugere-se que esta pesquisa seja replicada com vários motoristas em diversos trechos de estrada (conhecidos e desconhecidos ao motorista). E através destas, formar uma base de dados com intuito de conhecer profundamente as bases deste processo e, finalmente, firmar relações ótimas destas variáveis com o ato de dirigir. Desta forma, a tecnologia serviria como aliada na prevenção contra acidentes de trânsito.

Outro ponto importante a ser ressaltado é a ausência de ambientes que possam ser utilizados como restauradores psicofísicos em determinados trechos das estradas brasileiras. Toma-se como exemplo o trecho de Osório/RS até Porto Alegre/RS. Trecho que possui em torno de $100 \mathrm{~km}$ e em que não há postos de abastecimentos ou de conveniências disponíveis. Coincidentemente, este trecho foi considerado como um dos mais preocupantes e estressantes nesta pesquisa. Sugere-se que nos postos, os estacionamentos sejam pensados urbanisticamente, com a arborização de tais locais, privilegiando o silêncio, o ar fresco e saudável, permitindo que os motoristas possam ocupar esses locais e experimentar uma sensação de bem estar, que promova o relaxamento e a contemplação, de maneira que revigore as funções corpóreas e psicológicas, restaurando-as, para continuar a viagem. Ambientes, assim, permitem as pessoas readquiram seu controle de direção. Identificar essas qualidades restauradoras no trânsito, a fim de aplicá-las ao projeto das estradas, é um desafio importante a ser pensado, o que está diretamente ligado ao bem estar dos motoristas, e, sobretudo, evitando acidentes nas estradas.

\section{Referências bibliográficas}

AUBERT, A.E., SEPS B. \& BECKERS, F. Heart rate variability in athletes. Sports Med., 2003. 


\section{HUMANAS}

BERG, A. Preference for nature in urbanized societies: stress, restoration, and the pursuit of sustainability. Journal of Social Issues, 63: 79-96, 2007.

BERG, A., MAAS, J., VERHEIJ, R., \& GROENEWEGEN, P. Green space as a buffer between stressful life events and health. Social Science \& Medicine, 70: 1203-1210, 2010a.

CONSTANTINO, N.R.T. Novas funções do paisagismo: jardins terapêuticos. Anais do VII ENEPEA, 2004.

EVANS, G. A importância do ambiente físico. Psicologia USP, 16(1-2): 47-52, 2005.

GARCIA, F.S.F. Decorrências psicológicas de acidentes de trânsito em motoristas de transporte urbano de passageiros da Grande Florianópolis. Dissertação de Mestrado em Psicologia, Programa de Pós-Graduação em Psicologia. UFSC: Florianópolis, 2005.

GIULIANI, M.V. Proposta de resposta a partir da comparação entre "Environment Behavior" e "Journal of Environmental Psychology". Psicologia USP, 89-102, 2005.

GIULIANI, M.V. \& SCOPELLITI, M. Choosing restorative environments across the lifespan: A matter of place experience. Journal Environmental Psychology, 24: 423-37, 2004.

GODOY, M.F.; TAKAKURA, I.T.; \& CORREA, P.R. Relevância da análise do comportamento dinâmico não-linear (Teoria do Caos) como elemento prognóstico de morbidade e mortalidade em pacientes submetidos à cirurgia de revascularização miocárdica. Arq. Ciênc. Saúde, 2005.

GÜNTHER, H. A Psicologia Ambiental no Campo Interdisciplinar de Conhecimento. Psicologia USP, 2005.

GÜNTHER, H. Psicologia Ambiental e Psicologia do Trânsito - uma agenda de trabalho. Série: Textos de Psicologia Ambiental, No. 08). Brasília, DF: UnB. Laboratório de Psicologia Ambiental, 2004. Disponível em: www.unb.br/ip/lpa/ pdf/08PAePT.pdf. Acessado em: 14 de novembro de 2010.

HARTIG, T., BÖÖK, A., GARVILL, J., OLSSON, T. \& GÄRLING, T. Environmental influences on psychological restoration. Scandinavian Journal of Psychology: 378-93, 1996.

IVARSSON, C. \& HAGERHALL, C. The perceived restorativeness of gardens: Assessing the restorativeness of a mixed built and natural scene type. Urban Forestry \& Urban Greening, 7: 107-18, 2008 a. 
JOYE, Y. Architectural lessons from environmental psychology: the case of biophilic architecture. Review of General Psychology, 305-328, 2007.

KAPLAN, R. The nature of the view from home: psychological benefits. Environment and Behavior, 33: 507-42, 2001.

KAPLAN, R. \& KAPLAN, S. The Experience of Nature: a Psychological Perspective. Cambridge: Cambridge University Press, 1989.

KAPLAN, S. A Model of Person-Environment Compatibility. Environment and Behavior, 15: 311-22, 1983.

KARMANOV, D. \& HAMEL, R. Assessing the restorative potential of contemporary urban environment(s): Beyond the nature versus urban dichotomy. Science Direct: 115-25, 2008.

KJELLGREN, A. \& BUHRKALL, H. A comparison of the restorative effect of a natural environment with that of a simulated natural environment. Journal of Environmental Psychology, 2010.

KORT, Y.A.W. de; MEIJNDERS, A.L.; SPONSELEE, A.A.G. \& IJSSELSTEIJN, W.A. What's wrong with virtual trees? Restoring from stress in a mediated environment. Journal of Environmental Psychology, 2006.

KUHNEN, A. Interações humano-ambientais e comportamentos socioespacais. Interações pessoa-ambiente e saúde. São Paulo: Casa do Psicólogo, 2009.

LINDLEY, R.H.; WILSON, S.M.; SMITH, W.R. \& BATHURST, K. Reaction time and IQ: Shape of the task complexity function. Personality and Individual Differences, 1995.

MARÇOLLA, B.A. Os desafios da psicologia frente à questão ecológica: rumo à complexa articulação entre natureza e subjetividade. Psicologia: ciência e profissão, 22, 2002.

NISKANEN J.P.; TARVAINEN, M.P.; RANRA-AHO, P.O. \& KARJALAINEN, P.A. Software for advanced HRV analysis. Comput Methods Programs Biomed, 2004.

NOVAIS, L.D.; SAKABE, D.I.; TAKAHASHI, A.C.M.; GONGORRA, H.; TACIRO, C.; MARTINS, L.E.B.; OLIVEIRA, L.; SILVA, E.; GALLO Jr. \& CATAI, A.M. Avaliação da variabilidade da freqüência cardíaca em repouso de homens saudáveis sedentários e de hipertensos e coronariopatas em treinamento físico. Rev Bras Fisioter, 2004. 


\section{HUMANAS}

OLIVEIRA, A.C.F. de. Indicadores associados a acidentes de trânsito envolvendo motoristas de ônibus da cidade de Natal. Dissertação de Mestrado, Programa de Pós-Graduação em Psicologia. Natal: Universidade Federal do Rio Grande do Norte, 2003.

RIENER, A.; FERSCHA, A. \& ALY, M. Heart on the road: HRV analysis for monitoring a driver's affective state. Proceedings of the First International Conference on Automotive User Interfaces and Interactive Vehicular Applications. Essen, 2009.

ROCHA J.B.A. Infrações no trânsito: uma necessária distinção entre erros e violações. Interação em Psicologia, 2005.

SCHMIDT, R.A. \& CRAIG, A.W. Aprendizagem e performance motora: uma abordagem da aprendizagem baseada no problema. Porto Alegre: Artmed, 2001.

SILVA, F.H.V. de C. Medida da preditividade de instrumentos psicológicos nas avaliações psicológicas de condutores. Dissertação de Mestrado, Programa de Pós-Graduação em Psicologia. Natal: Universidade Federal do Rio Grande do Norte, 2008.

TAKASE, E. Interações humano-ambientais: as contribuições da neurociência. Interações pessoa-ambiente e saúde. São Paulo: Casa do Psicólogo, 2009.

TASSARA, E.T.O. Psicologia ambiental e futuro: reflexões geopolíticas sobre política ambiental. Psicologia USP: 261-67, 2005.

UZZELL, D. Questionando os métodos na pesquisa e na prática interdisciplinar da psicologia ambiental. Psicologia USP: 185-99, 2005.

VANDERLEI, L.C.M.; PASTRE, C.M.; HOSHI, R.A.; CARVALHO, T.D. \& GODOY, M.F. Noções básicas de variabilidade da freqüência cardíaca e sua aplicabilidade clínica. Revista Brasileira de Cirurgia Cardiovascular, 24 Edição, 2009.

VELARDE, M.D.; FRY, G. \& TVEIT, M. Health effects of viewing landscapes: Landscape types in environmental psychology. Urban Forestry \& Urban Greening, 6: 199-212, 2007. 